\title{
A 3-D look into the atmosphere?
}

\author{
G. A. Wade ${ }^{1}$, L. Mashonkina ${ }^{2}$, T. Ryabchikova ${ }^{2}$, J. Krticka ${ }^{3}$, J.
} Silvester $^{1}$, O. Kochukhov ${ }^{4}$, J. C. Sousa ${ }^{5}$, T. Nomura ${ }^{6}$, G. M. Wahlgren $^{7}$, M. Gruberbauer ${ }^{8}$, S. Hubrig ${ }^{9}$, M. Briquet ${ }^{10}$, K. Yüce ${ }^{11}$, N. Drake ${ }^{12}$, N. Nunez ${ }^{13}$, R. O. Gray ${ }^{14}$, J. Ziznovsky ${ }^{15}$, C. Cowley ${ }^{16}$, A. Shavrina ${ }^{17}$, M. Dworetsky ${ }^{18}$, O. Pintado ${ }^{19}$, N. Polosukhina ${ }^{20}$ \&

\author{
L. Cidale ${ }^{21}$ \\ ${ }^{1}$ Royal Military College, Canada \\ ${ }^{2}$ Institute of Astronomy, Russian Academy of Sciences, Russia \\ ${ }^{3}$ Masaryk University, Czech Republic \\ ${ }^{4}$ Uppsala University, Sweden \\ ${ }^{5}$ CAUP-Centro de Astrofisica da Universidade do Porto, Portugal \\ ${ }^{6}$ University of Tokyo, Japan \\ ${ }^{7}$ NASA-GSFC/CUA, USA \\ ${ }^{8}$ St Mary's University, Canada \\ ${ }^{9}$ Astrophysikalisches Institut Potsdam, Germany \\ ${ }^{10}$ Instituut voor Sterrenkunde, Katholieke Universiteit Leuven, Belgium \\ ${ }^{11}$ Ankara University, Turkey \\ ${ }^{12}$ Sobolev Astronomical Institute of St. Petersburg State University, Russia \\ ${ }^{13}$ Casleo-UNSJ, Argentina \\ ${ }^{14}$ Appalachian State University, USA \\ ${ }^{15}$ Astronomical Institute of the Slovak Academy of Sciences, Slovakia \\ ${ }^{16}$ Dep. of Astronomy, University of Michigan, USA \\ ${ }^{17}$ Main Astronomical Observatory, National Academy of Sciences of Ukraine \\ ${ }^{18}$ University College London, United Kingdom \\ ${ }^{19}$ Instituto Superior de Correlación Geológica-CONICET, Argentina \\ ${ }^{20}$ Crimean Astrophysical Observatory, Ukraine \\ ${ }^{21}$ Faculdad de Ciencias Astronomicas y Geofisicas, Argentina
}

\begin{abstract}
The atmospheres of chemically peculiar stars can be highly structured in both the horizontal and vertical dimensions. While most prevalent in the magnetic stars, these structures can also exist in non-magnetic stars. In addition to providing an important window to understanding the physical processes at play in these complex atmospheres, they can also be exploited to study stellar pulsations. This article reviews contributions to the session "A 3D look into the atmosphere" of the Joint Discussion "Progress in understanding the physics of Ap and related stars". It is divided into 3 sections: "Magnetic field and surface structures", "Pulsations in the atmospheres of roAp stars/inversions", and "Spectral synthesis/atmospheric models".
\end{abstract}

Keywords. stars: chemically peculiar, stars: atmospheres, stars: variables: roAp stars, stars: magnetic fields

\section{Magnetic field and surface structures}

Magnetic field. Following their discovery by Babcock (1947), strong, organised magnetic fields have been found to exist in a small fraction (e.g. Shorlin et al. 2002, Power 
et al. 2008) of main sequence intermediate-mass A and B stars (the "Ap/Bp stars"). Historically, these fields have been modeled as "oblique rotators": strong, long-lived dipoles or aligned low-order multipoles, frozen into the stellar plasma, inclined relative to the stellar rotation axis, and rotating rigidly with the star. These properties, along with a lack of any strong, positive correlation of magnetic field strength with rotation or mass, has led most investigators to suppose that these fields are "fossils": the slowly-decaying remnants of magnetic field accumulated from the ISM during star formation, or generated by a pre-main sequence dynamo. Recent studies of these fields have yielded important new information about their detailed properties. Detailed mapping using the Magnetic Doppler Imaging method (Kochukhov \& Piskunov 2002) has revealed the presence of complex structures in the photospheric magnetic fields of 53 Cam (Kochukhov et al. 2004a) and $\alpha^{2}$ CVn (Kochukhov \& Wade, in preparation), the origin of which is unknown. Silvester (this conference, JD04-o:4) reported on a continuation of this work using new measurements acquired using the ESPaDOnS and Narval instruments at the CanadaFrance-Hawaii Telescope and Télescope Bernard Lyot, respectively. Aurière et al. (2007) obtained magnetic field measurements of $\sim 30 \mathrm{Ap} / \mathrm{Bp}$ stars with weak or previouslyundetected magnetic fields, demonstrating that each of them hosted a magnetic field stronger than about $300 \mathrm{G}$. They interpreted this apparent field strength threshold as a "critical field strength", below which magnetic fields are unstable to rotational distortion. Lignières et al. (2009) reported the detection of a circular polarisation signature in the mean Least-Squares Deconvolved spectral line of Vega. Interpreting this as evidence of a weak magnetic field, they proposed that Vega may be the first of a new class of magnetic A-type stars - perhaps those stars in which the initial fossil fields were weaker than the "critical field strength", and have hence decayed.

Meanwhile, intensive investigations of pre-main sequence Herbig Ae/Be stars has yielded the discovery of magnetic stars at this early phase of evolution which exhibit field properties very similar to those of Ap stars (e.g. Alecian et al. 2008, 2009, also Alecian's presentation at this conference, JD04-o:11), further supporting the fossil field hypothesis. At the other end of the main sequence, Bagnulo et al. (2006) and Landstreet et al. $(2007,2008)$ used samples of Ap stars observed in open clusters to demonstrate a dramatic decrease with age of the ensemble-averaged magnetic field strength of Ap stars - a phenomenon that does not appear to be fully explainable by stellar expansion during main sequence evolution. Finally, recent detections of variable Zeeman signatures in mean spectral lines of intermediate-mass red giant stars (e.g. Aurière et al. 2008, 2009) have allowed a first insight into the post-main sequence evolution of Ap stars, and potentially the origin of the magnetic fields observed in some white dwarfs.

At this conference, posters by Hubrig et al. (JD04-p:4) and Drake et al. (JD04-p:9) discussed the magnetic properties of some individual stars with strong magnetic fields.

Surface structures. The presence of strong lateral chemical nonuniformities in the atmospheres of magnetic Ap/Bp stars has long been known. Since the pioneering work in the 1970s and 80s (see Khokhlova (1974), and works by Piskunov, Rice, Hatzes) to develop techniques to "map" these structures by modeling the resultant variations of rotationally-broadened line profiles, the technique of "Doppler Imaging" has evolved significantly. Investigations such as that of Kochukhov et al. (2004b) of the (magnetic) rapidly oscillating Ap (roAp) star HR 3831 report maps of the magnetic field and the abundances of many chemical species, demonstrating a tremendous diversity of abundance patterns and suggestive relationships with the magnetic field and rotational geometry. Kochukhov (this conference, JD04-o:3) reported results of 3-dimensional mapping of the atmospheric chemical structures of the Ap star $\theta$ Aur: maps of not only the surface distribution of Fe, but also the vertical stratification of this element. These authors 


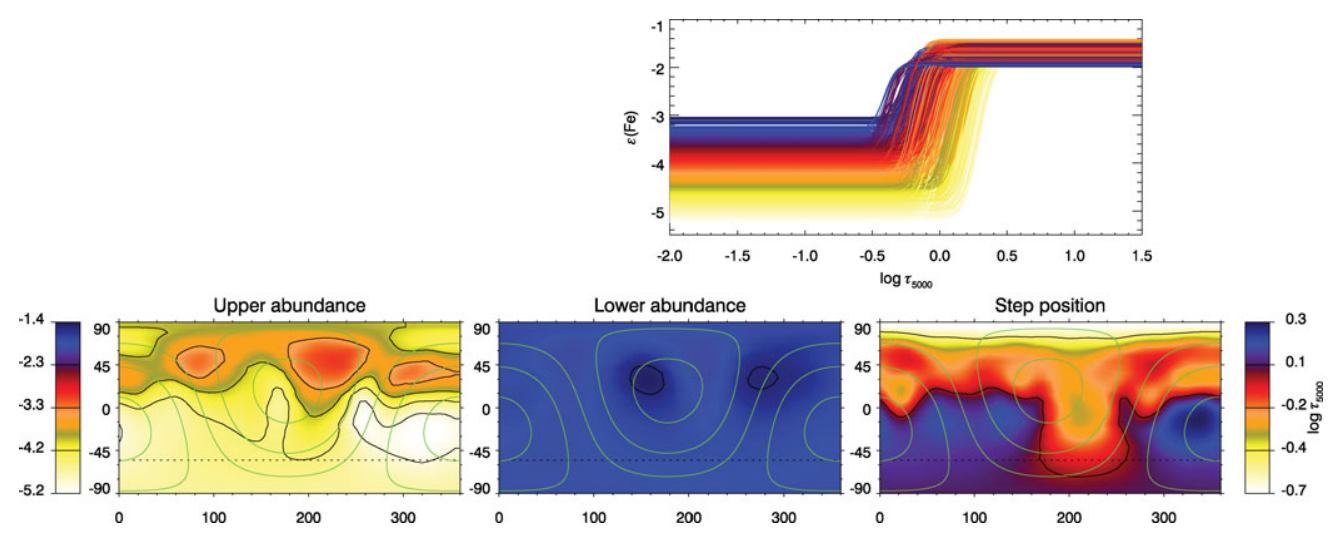

Figure 1. 3-D Doppler imaging reconstruction of the Fe distribution in the atmosphere of magnetic Ap star $\theta$ Aur (HD 40312). The vertical abundance profile for each surface element is given by a step function, characterized by the Fe abundance in the upper and lower atmospheric layers as well as the position of transition region. The bottom panel shows reconstructed maps for these three parameters. The upper panel displays all vertical abundance profiles plotted on top of each other and color-coded according to the upper abundance.

discovered that while the abundance of Fe in the lower atmosphere is not strongly correlated with the presence of spots, the abundance in the upper atmosphere and the position of the lower/upper atmosphere transition depth are. Their results are illustrated in Fig. 1. Krticka (this conference, JD04-o:1) demonstrated for the Bp star HD 7224 that the photometric variability could be acceptably explained by the opacity variations introduced by the nonuniform distributions of $\mathrm{Si}$ and $\mathrm{Fe}$ in its atmosphere. A poster by Shavrina et al. (JD04p:21) examined the presence of Li spots on the roAp star HD 12098, while a poster of Polosukhina et al. (JD04-p:27) discusses a connection of the Li spots on the surfaces of Ap stars with the magnetic field configuration.

A remarkable decade-old discovery by Ryabchikova et al. (1999) that has now been confirmed in several stars (Adelman et al. 2002, Kochukhov et al. 2005, Hubrig et al. 2006 ) is the presence of nonuniform distributions of $\mathrm{Hg}$ and other elements in atmospheres of some relatively rapidly-rotating HgMn stars. Sensitive searches for magnetic fields in these objects (e.g. Wade et al. 2006) have yielded no detections at the level of a few $\mathrm{G}$, suggesting that the origin of these spots is not a magnetic field, but rather some other dynamical effect (e.g. rotation, and/or presence within a close binary). At this conference, posters by Nunez et al. (JD04-p:12) and Briquet et al. (JD04-p:5) focused on this phenomenon.

\section{Pulsations in the atmospheres of roAp stars / inversions}

An explicit review on pulsations in the atmospheres of chemically peculiar magnetic stars is presented recently by Kochukhov in 2009, and references therein. Here we briefly review the main characteristics of the rapidly oscillating Ap (roAp) stars.

General characteristics of roAp stars. A group of the roAp stars represents the coolest magnetic Ap stars and occupies the central part of the main sequence band in the effective temperature range $6300-8100 \mathrm{~K}$. On the HR diagram roAp stars overlap with other groups of pulsating stars - $\delta$ Sct-type and solar-like pulsators - however the nature of the pulsations and their characteristics are different. roAp stars pulsate in high-overtone, 
low-degree p-modes with typical periods between 4 and 22 min, photometric amplitudes of $0.2-10 \mathrm{mmag}$ and radial velocity (RV) amplitudes ranging from $3 \mathrm{~m} \mathrm{~s}^{-1}$ ( $\beta \mathrm{CrB}-$ Hatzes \& Mkrtichian 2004) to $5 \mathrm{~km} \mathrm{~s}^{-1}$ (HD 99563 - Elkin et al. 2005). At present, the roAp group includes 38 members. Thirty-two roAp stars were discovered by ground-based photometric monitoring (see a review by Kurtz \& Martinez 2000), and six roAp stars (HD 75445, HD 115226, HD 116114, $\beta$ CrB, HD 154708, HD 218994) were discovered using high-resolution time-series spectroscopy during the last five years. Spectroscopic RV observations of 22 roAp stars suggest a possible anti-correlation of RV amplitudes with the magnetic field strength: the stronger the field, the lower the RV amplitude. However, statistics are sparse, and this result requires confirmation.

Atmospheric pulsations. An outstanding characteristic of RV variations in roAp stars is a diversity of pulsation signatures in spectral lines of different atoms/ions. In the same star one can measure RV amplitudes from few $\mathrm{ms}^{-1}$ (Fe lines) to hundreds of $\mathrm{ms}^{-1}$ (rare-earth elements lines - REE, see Ryabchikova et al. 2007). This phenomenon was explained as a propagation of the acoustic pulsation wave in a chemically-stratified stellar atmosphere (Ryabchikova et al. 2002), where stratification results from chemical diffusion in a globally-stable atmosphere. In most roAp stars for which detailed line-by-line pulsation analysis has been performed, pulsations have a standing- or running wave character with an increase of RV amplitudes towards the outer layers. In two stars, $33 \mathrm{Lib}$ (Mkrtichian et al. 2003) and 10 Aql (Sachkov et al. 2008) a definite presence of the pulsation node at the position of REE line formation layers at $\log \tau_{5000}=-4$ is observed with the opposite direction of the phase jumps. It looks like in $33 \mathrm{Lib}$ we have pulsational inversion: instead of the outwardly-running wave observed in most other stars, an inwardly-propagating wave exists. According to Sousa \& Cunha (2008, JD04_o:7) this behaviour as well as the appearance of the nodes may be interpreted as the superposition of magnetic standing and acoustic running waves in the atmospheres of magnetic stars, mimicking an inwardly-propagating wave, node layers, etc., that depends on the magnetic field geometry and on the observer's perspective. Khomenko \& Kochukhov (2009) came to the same conclusion in their 2D magneto-hydrodynamical simulations of magneto-acoustic pulsations in realistic atmospheres of roAp stars. They considered the propagation of pulsation waves with initial amplitude $100 \mathrm{~m} \mathrm{~s}^{-1}$, excited by a radial pulsation mode below the visible surface of the realistic atmospheric model $\left(T_{\text {eff }}=7750\right.$ $\mathrm{K}, \log \mathrm{g}=4.0$ ) with a constant inclined magnetic field. MHD simulations confirmed the theoretical results by Saio (2005) about the absence of pulsational magnetic field variations above $1 \mathrm{G}$. On the other hand, MHD simulations showed significant pulsational variations of the thermodynamic parameters temperature and density at different layers of the stellar atmosphere, in particular at the photospheric base where the density inversion takes place.

In some roAp stars the frequency spectrum of photometric variations differs from that measured from spectroscopic variations. Kurtz et al. (2006) found a 'new type' of upper atmospheric pulsations. In some stars such as 33 Lib and HD 134214, new frequencies - not observed in photometry - appear in the RV variations of Pr III-Nd III lines. These lines are formed in the upper atmospheric layers; hence, new frequencies are either amplified or even excited in these layers. Extensive photometric + spectroscopic studies are required to understand this phenomenon.

Nomura \& Shibahashi (JD04_o:14) presented first results on numerical simulations of line profile variations in roAp stars. Their simplified model could reasonably explain the observed bisector variations in the $\mathrm{H} \alpha$ core in all roAp stars (an increase of RV amplitudes with the depth of the core), while it failed to explain the observed core-to-wing bisector variations in $\mathrm{Nd}$ III lines. $\mathrm{H} \alpha$ core bisector variations agree with the general picture of 


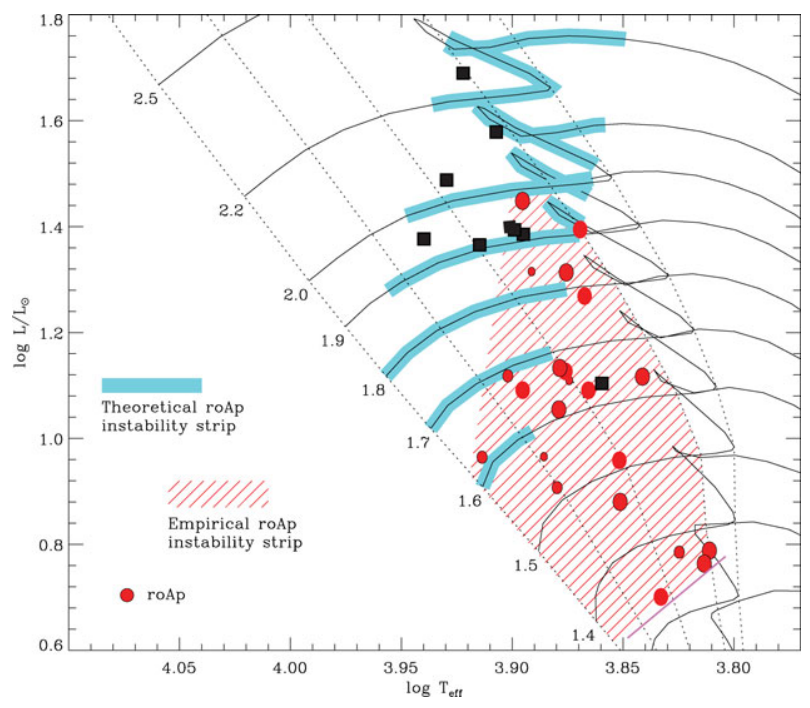

Figure 2. Theoretical and observed IS for roAp stars. Positions of the roAp stars are shown by filled circles with size proportional to parallax value, while filled squares indicate positions of the nine luminous Ap stars in which pulsations were not found.

acoustic wave propagation in the stellar atmosphere - an increase of RV towards the upper layers. Contrary to the $\mathrm{H} \alpha$ core, in most of stars we observe a decrease of the RV amplitude with the depth within the Nd III line profile.

Evolutionary status of roAp stars. The current theory of pulsations in roAp stars proposes that they are excited by a $\kappa$-mechanism acting in the hydrogen ionization zone. A globally-organized magnetic field effectively suppresses convection and makes excitation possible (Balmforth et al. 2001). Abundance gradients, in particular helium settling in the stable atmospheres due to diffusion, also favour the hydrogen $\kappa$-mechanism (Vauclair \& Théado 2004). However, first calculations of the instability strip (IS) of roAp stars (Cunha 2002) showed that the red border is shifted to higher luminosities than is revealed by observations. Fig. 2 taken from Kochukhov (JD04_p:2) presents the position of the roAp stars with known Hipparcos parallaxes on the HR diagram. The theoretical IS is taken from the recent calculations by Théado et al. (2009) who considered the effect of metallicity on the pulsational excitation in roAp stars. These calculation do not differ significantly from Cunha's calculations. We also plot nine luminous Ap stars in which pulsations are predicted theoretically, but were not found in spectroscopy (Freyhammer et al. 2008). It is evident that the theoretical and observed IS are shifted both in luminosity and in effective temperature relative each other, which means that the currently accepted excitation mechanism model needs to be improved.

Modelling the pulsations in individual stars. Pulsation modelling of two rather rapidly rotating roAp stars HD 83368 (HR 3831 - Kochukhov 2004, 2006) and HD 99563 (Freyhammer et al. 2009) based on spectroscopic time-series observations obtained over the rotational period shows a single mode which is a distorted oblique dipole mode. First indirect imaging of nonradial pulsations in the roAp star HD 83368 by Kochukhov (2004) directly demonstrates an alignment of pulsations with the axis of the dipolar-like global magnetic field, thus proving the oblique pulsator model proposed by Kurtz (1982) to explain rotational modulation of the photometric pulsation amplitudes in roAp stars.

Frequency modelling of slowly rotating roAp stars are based on long-time photometric ground-based (HD 24712 - WET campaign) or space-based ( $\gamma$ Equ, 10 Aql - MOST; 


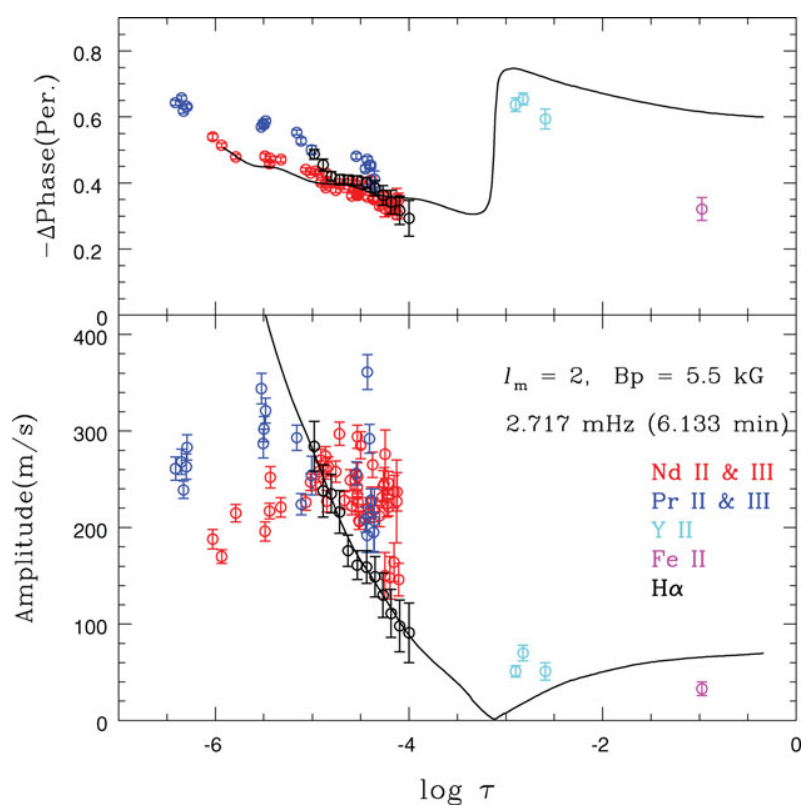

Figure 3. Theoretical (black line) and observed (open circles) pulsational RV phase and amplitude distributions in the atmosphere of the roAp star HD 24712.

$\alpha$ Cir - WIRE) observations. Only for HD 101065 (Przybylski's star) frequency modelling was based on long-duration spectroscopic monitoring. The frequency pattern in these stars is richer and can usually be represented by a combination of odd-even modes with $\ell=0-3$ (see Gruberbauer et al. 2008 for $\gamma$ Equ; Huber et al. 2008 for 10 Aql; Bruntt et al. 2009 for $\alpha$ Cir; and Mkrtichian et al. 2008 for HD 101065). HD 24712 is the first star where not only the frequency pattern derived by Kurtz et al. (2005) in WET campaign, but also RV amplitude and phase distributions for the two highest amplitude frequencies in the atmosphere were modelled (see Saio et al. JD11_p:16). An improved model atmosphere of HD 24712 which takes into account chemical anomalies as well as abundance stratification for a few of the most important elements - Si, Ca, Cr, Fe (Shulyak et al. 2009, see also next Section) - was used in the pulsation modelling. Magnetic fields are required to fit the observed frequencies in all models, with magnetic perturbations usually 2-3 times larger than actually observed. The only exception is HD 24712, where the polar intensity of the magnetic dipole, $B_{\mathrm{p}} \approx 5.5 \mathrm{kG}$, needed to represent the observed pulsation data, is roughly consistent with the observed value of $4.2 \mathrm{kG}$. Fig. 3 taken from Saio et al. (JD11_p:16) shows a comparison between the observed and theoretical distributions of the pulsational RV amplitudes and phases in the atmosphere of HD 24712 for the main highest amplitude frequency.

Formally, the modelling is successful, however for all models of HD 101065 and HD 24712 no high-order p-modes appropriate to the observed oscillations are excited. This is also the case for few modes in the $\gamma$ Equ model. These negative results agree with the predicted roAp IS positions for these stars. These stars are too cool for p-modes to be excited by the $\kappa$-mechanism in the hydrogen ionization zone. The temperature inversion in the atmosphere of HD 24712 (Shulyak et al. 2009) seems too small to help excite high-order p-modes. 


\section{Spectral synthesis / atmospheric models}

In this section, we highlight recent results on modelling of the atmospheres and spectra of chemically peculiar (CP) stars with non-uniform vertical distributions of elements, and describe advances in searching for and investigating exotic chemical elements in CP stars.

Self-consistent diffusion model atmospheres. The microscopic diffusion process is believed to be the main cause of chemical stratification in stellar atmospheres. LeBlanc et al. (2009) performed self-consistent calculations of atmospheric structure and element diffusion, yielding the abundance gradients for 39 elements ( $\mathrm{H}-\mathrm{Ga}, \mathrm{Kr}-\mathrm{Nb}$, Ba, and La). No competing physical processes, including mass loss, convection, and turbulence, were considered. In the model with $T_{\text {eff }}=8000 \mathrm{~K}$ and $\log \mathrm{g}=4$, diffusion produces a highly non-uniform distribution of every included element with a lower abundance in the upper layers, above $\log \tau_{5000}=-1$, and an abundance increase in deep layers, $-1 \leqslant$ $\log \tau_{5000}<0$. The change in temperature due to stratification is small in the continuum and line-forming region, up to $5 \%$. LeBlanc et al. (2009) found that the effect of diffusion depends strongly on effective temperature. In the hotter model with $T_{\text {eff }}=12000 \mathrm{~K}$, the abundance gradient for each included element is small and the calculated abundance exceeds the initial one throughout the atmosphere. Depending on the treatment of the redistribution of momentum among the various ions of an element, the temperature in the continuum and line-forming region can be up to $10 \%$ higher compared to that in a chemically homogeneous model with $10 \times$ enhanced abundances of metals. The accuracy of the self-consistent diffusion models is defined by the accuracy and completeness of atomic data, in particular transition probabilities and photoionization cross-sections for a variety of elements. For most heavy elements beyond the iron group, the required data is either incomplete or missing.

The self-consistent diffusion model atmospheres are only just beginning to be utilized in the analysis of CP stars. The theoretical results support observational findings about the abundance jumps for the Fe group and lighter metals in cool Ap stars. For chromium and iron in cool Ap stars, Ryabchikova (2008) highlighted an agreement between the predicted and empirical abundance profiles for the position and the magnitude of the abundance jumps. At this conference, Ryabchikova et al. (JD04_p:13) showed much smaller abundance gradients for the hotter star HD $170973\left(T_{\text {eff }}=11200 \mathrm{~K}\right)$ in agreement with the prediction of the diffusion theory.

For $T_{\text {eff }}=8000 \mathrm{~K}$ and $\log \mathrm{g}=4$, LeBlanc et al. (2009) calculated a self-consistent diffusion model atmosphere with a horizontal magnetic field of $2 \mathrm{kG}$ and found that diffusive motions of the ionized species are efficiently impeded in the upper atmosphere and metals are pushed outwards. In the layers around $\log \tau_{5000}=-3$, the temperature increases by approximately $10 \%$ compared to that in the chemically homogeneous model.

Alecian \& Stift (2007) calculated the stratification of some metals (Mg, Si, Ca, Ti, and $\mathrm{Fe}$ ) in the models with fixed atmospheric structure and magnetic fields of up to $10 \mathrm{kG}$ and various inclination. They found that (i) the differences in abundance profiles between the non-magnetic and magnetic with vertical field atmospheres are small, (ii) the role of magnetic field is small for deep layers, below $\log \tau_{5000}=-1$, independently of the field inclination, and (iii) the horizontal field can be very efficient in helping the accumulation of elements in the upper atmosphere.

Non-local thermodynamic equilibrium (NLTE) line formation for Pr II-III and Nd IIIII in A-type stars. The rare-earth elements play a key role in understanding the phenomenon of Ap stars. Classic LTE analysis finds a strong violation of the ionization equilibrium between the first and second ions of the REE in the atmosphere of roAp stars suggesting a non-uniform vertical distribution of these elements in the atmosphere. 
Contrary to the iron group and lighter metals, the REE are, probably, accumulated in the uppermost atmospheric layers, where the LTE assumption is not valid. The difficulties of the statistical equilibrium calculations for the REE are due to incompleteness of the system of known energy levels and poorly known photoionization and collisional data. Mashonkina et al. $(2005,2009)$ constructed comprehensive model atoms for Nd II-III and Pr II-III using not only measured but also calculated energy levels. The calculations for model atmospheres with uniform distributions of elements showed that NLTE acts in the right direction and tends to reduce the abundance difference between the first and second ions, but fails to remove this disparity completely. For example, for the benchmark roAp star HD $24712\left(T_{\text {eff }}=7250 \mathrm{~K}\right)$, the difference in LTE abundances derived from the Pr III and Pr II lines amounted 2.1 dex, and only a 0.2 dex smaller difference was obtained in the NLTE case. The left panel of Fig. 4 illustrates the NLTE effects on the Pr II-III lines in HD 24712 when the distribution of praseodymium is assumed to be uniform with $[\mathrm{Pr} / \mathrm{H}]=3$.

Mashonkina et al. $(2005,2009)$ then checked various abundance profiles, independently for praseodymium and neodymium, trying to fit the observed equivalent widths of the lines of both ionization stages in HD 24712 with a single abundance distribution. It was found that both elements are concentrated in the uppermost atmospheric layers, above $\log \tau_{5000}=-4$, with abundance enhancements of 4.5 dex for neodymium and 5 dex for praseodymium compared to the corresponding solar abundances. For comparison, the praseodymium and neodymium enriched layer has to be located above $\log \tau_{5000}=$ -8 when stratification analysis is performed under LTE conditions. In the stratified atmosphere, the calculated NLTE effects are very large (Fig. 4) because the line formation occurs in the high atmosphere, where collisions are rare and inefficient in establishing thermodynamic equilibrium. The lines of Pr II and Nd II are strongly weakened compared to their LTE strengths, and the opposite effect is found for Pr III and Nd III. The NLTE abundance corrections may reach +1.2 dex for the first ions and -0.7 dex for the second ions.

Stratified model atmospheres with empirical abundance profiles. Kochukhov et al. (2009) and Shulyak et al. (2009) suggested the method of self-consistent analysis, where the individual abundances and chemical stratification derived for a given star are then used to recalculate its model atmosphere structure. The entire process of determining stellar parameters, chemical abundances and stratification and calculation of the model atmosphere has to be repeated until convergence is achieved. Analysis of two roAp stars with similar stellar parameters, HD $24712\left(T_{\text {eff }} / \log g=7250 \mathrm{~K} / 4.1\right.$, Shulyak et al. 2009) and
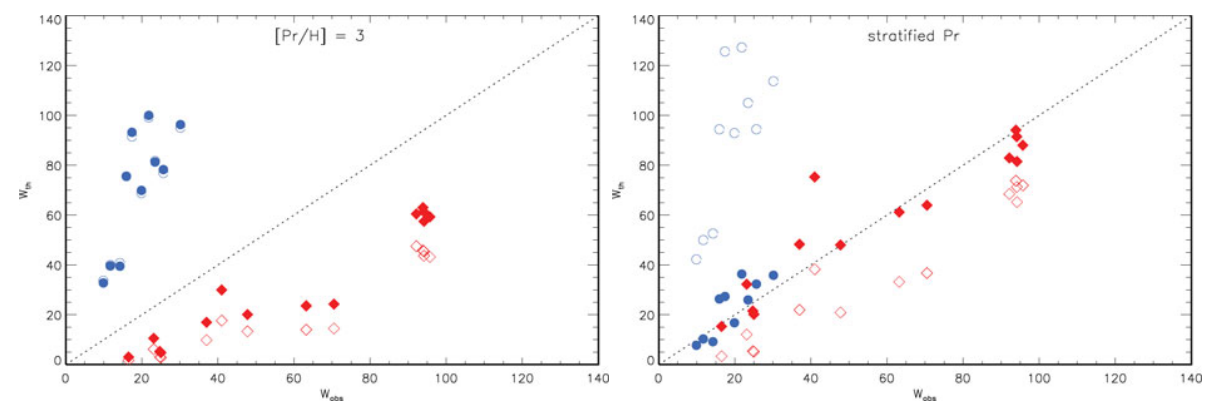

Figure 4. Observed equivalent widths of the Pr II (circles) and Pr III (diamonds) lines in HD 24712 compared with the theoretical non-LTE (filled symbols) and LTE (open symbols) equivalent widths for the uniform $\mathrm{Pr}$ distribution with $[\mathrm{Pr} / \mathrm{H}]=3$ (left panel) and the stratified Pr distribution (right panel). 
$\alpha$ Cir $(7500 \mathrm{~K} / 4.1$, Kochukhov et al. 2009), showed that the calculated atmospheric structure depends on the individual abundance profiles. For $\alpha$ Cir, four elements, Si, Ca, $\mathrm{Cr}$ and $\mathrm{Fe}$, were allowed to have non-uniform vertical distributions. It was found that all of them are concentrated in the lower atmosphere and this leads to a backwarming effect. The corresponding reduction of effective temperature derived from the Balmer line wings amounted to $200 \mathrm{~K}$.

For HD 24712, stratification of the same four elements and, in addition, that of Sr, $\mathrm{Ba}, \mathrm{Pr}$, and Nd, was taken into account. The abundance profiles for praseodymium and neodymium were derived from the NLTE analysis of the Pr II-III and Nd II-III lines. The backwarming effect was found to be weaker in HD 24712 compared to that in $\alpha$ Cir due to lower abundances of chromium and iron. The accumulation of praseodymium and neodymium above $\log \tau_{5000}=-4$ caused a temperature increase of up to $600 \mathrm{~K}$ in these same layers. It is worth noting that the changes in temperature structure due to stratification of praseodymium and neodymium did not affect the formation of the Balmer line wings and the visible continuum and did not result in any change of the stellar parameters of HD 24712.

For both stars, the differences between initial and final abundance profiles were found to be within the error bars of the determination of stratification. It should be stressed that the stellar parameter range addressed in the studies of Kochukhov et al. (2009) and Shulyak et al. (2009) is very narrow, and the validity of their findings should not be extended out of this range.

Exotic elements in CP stars. Progress in extending our knowledge of spectrum anomalies was made thanks to laboratory measurements of atomic data, calculations of atomic structures, and high-resolution spectral observations. At this conference, Wahlgren et al. (JD04_o:9) found nearly all lanthanides and also $\mathrm{Pt}, \mathrm{Au}, \mathrm{Hg}$, and $\mathrm{Bi}$ in the ultra-violet spectrum of the spectrum transition star HR 3383. Cowley et al. (JD04_p:17) first investigated the spectra of Nb II, Re II, and Os II in the spectrum of the star HD 65949. Dworetsky \& Maher-McWilliams (JD04_p:20) determined the gold abundance in nine HgMn stars. Cowley et al. (JD04-p:22, see also Cowley et al. MNRAS, 396, 485, 2009) gave evidence for isotope separation of calcium in the atmosphere of some CP stars from measurements of differences in the wavelengths of the cores and the wings of the infrared triplet of Ca II. Ryabchikova et al. (JD04_p:8) considered an effect of the completeness of the REE line data on the atmospheric structure calculations and spectral synthesis of the extreme peculiar star Przybylski's star, and concluded that the lack of these data may lead to mistaken identification of actinides in cool REE-rich stars.

\section{Acknowledgements.}

ML, ND, and TR acknowledge partial support from the International Astronomical Union for participation at the IAU XXVII General Assembly. ML and TR also thank the Russian Foundation for Basic Research (grants 09-02-08-244-z, 09-02-00002-a, 08-0200469-a) and the Russian Federal Agency on Science and Innovation (No. 02.740.11.0247). GAW is supported by the Natural Science and Engineering Council of Canada (NSERC) and the Department of National Defence (Canada). JS is supported in part by NSERC Discovery Grants held by GAW and by David Hanes (Queen's University). KY's participation was supported by an IAU Travel Grant and by Ankara University. OK is a Royal Swedish Academy of Sciences Research Fellow supported by a grant from the Knut and Alice Wallenberg Foundation.

\section{References}

Adelman S. J., Gulliver A., Kochukhov O., \& Ryabchikova T. 2002, ApJ 575, 449 
Alecian E., Catala C., Wade G. A., Donati J.-F.., et al. 2008, MNRAS 385, 391

Alecian E., Wade G. A., Catala C., Bagnulo S., et al. 2009, MNRAS 400, 354

Alecian, G. \& Stift, M. J. 2007, A\&\&A, 475, 659

Aurière M., Wade G. A., Silvester J., Lignières F. et al., 2007, A $6 A$ 475, 1053

Aurière M., Konstantinova-Antova R., Petit P., Charbonnel C., et al.2008, A $\& A$ 491, 499

Aurière M., Wade G. A., Konstantinova-Antova R., Charbonnel C., et al. 2009, A\&A 504, 231

Babcock, H. W. 1947, ApJ 105, 105

Bagnulo S., Landstreet J. D., Mason E., \& Andretta V. 2006, A\& A 450, 777

Balmforth, N. J., Cunha, M. S., Dolez, N., et al. 2001, MNRAS, 323, 362

Bruntt, H., Kurtz, D. W., \& Cunha, M. S. 2009, MNRAS, 396, 1189

Cunha, M. S. 2002, MNRAS, 333, 47

Elkin, V. G., Kurtz, D. W., \& Mathys, G. 2005, MNRAS, 364, 864

Freyhammer, L. M., Kurtz, D. W., Cunha, M. S., et al. 2008, MNRAS, 385, 1402

Freyhammer, L. M., Kurtz, D. W., Elkin, V.G., et al. 2009, MNRAS, 396, 325

Gruberbauer, M., Saio, H., Huber, D., et al. 2008, A\&SA, 483, 239

Hatzes, A.P. \& Mkrtichian, D. E. 2004,MNRAS, 351, 663

Huber, D., Saio, H., Gruberbauer, M., et al. 2008, A\& $A, 480,223$

Hubrig, S., Gonzalez, J. F., Savanov, I., \& Schöller, M. 2006, MNRAS, 379, 1953

Khokhlova V. 1974, Nauchn. Inf., Vyp., 33, 11

Khomenko, E. \& Kochukhov, O. 2009, ApJ, 704, 1218

Kochukhov, O., Piskunov, N., Sachkov, M., \& Kudryavtsev, D. 2005, A\&A 4 439, 1093

Kochukhov, O. \& Piskunov, N. 2002, A\&A, 388, 868

Kochukhov, O. 2004, ApJ, 615, L49

Kochukhov, O., Bagnulo S., Wade G.A., Sangalli L., et al. 2004a, A\&A 414, 613

Kochukhov, O., Drake, N. A., Piskunov, N., \& de la Reza, R. 2004b, A\&\&A 424, 935

Kochukhov, O. 2006, A\&\&A, 446, 1051

Kochukhov, O. 2009, CoAst, 159, 61

Kochukhov, O., Shulyak, D., \& Ryabchikova, T. A. 2009, A\&A 499, 851

Kurtz D. W. 1982, MNRAS 200, 807

Kurtz, D. W. \& Martinez, P. 2000, Baltic Astronomy 9, 253

Kurtz, D. W. Cameron, C., Cunha, M. S. et al. 2005, MNRAS 358, 651

Kurtz, D. W. Elkin, V. G., \& Mathys, G. 2006, MNRAS 370, 1274

LeBlanc, F., Monin, D., Hui-Bon-Hoa, A., \& Hauschildt, P. H. 2009, A\&̈A, 495, 937

Landstreet J. D., Bagnulo S., Andretta V., Fossati L., et al. 2007, A $\mathscr{E} A$ 470, 685

Landstreet J. D., Silaj J., Andretta V., Bagnulo S., et al. 2008, A\&\&A 481, 465

Lignières F., Petit P., Bohm T., \& Aurière M. 2009, A $\mathscr{E} A$ 500, 41

Mashonkina, L., Ryabchikova, T. A., \& Ryabtsev, A. N. 2005, A\&\&A 441, 309

Mashonkina, L., Ryabchikova, T. A., Ryabtsev, A. N., \& Kildiyarova, R. 2009, A\&A 495, 297

Mkrtichian, D. E., Hatzes, A. P., \& Kanaan, A. 2003, MNRAS 345, 781

Mkrtichian, D. E., Hatzes A. P., Saio, H., \& Shobbrook, R. R. 2008, A\&广A 490, 1109

Power J., Wade G. A., Aurière M., Silvester J., \& Hanes D. 2008, Cont. Ast. Obs. Skalnaté Pleso 38, 443

Ryabchikova, T. A., Malanushenko, V. P., \& Adelman, S. J. 1999, A $\& A$ A 351, 963

Ryabchikova, T., Piskunov, N., Kochukhov, O., et al. 2002, A\&A 384, 545

Ryabchikova, T., Sachkov, M., Kochukhov, O., \& Lyashko, D. 2007, A\&A 473, 907

Ryabchikova, T. 2008, Contr. Astron. Obs. Skalnaté Pleso 38, 257

Sachkov, M., Kochukhov, O., Ryabchikova, T., et al. 2008 MNRAS 389, 903

Saio, H. 2005, MNRAS, 360, 1022

Shorlin S. L. S., Wade G. A., Donati J. F, Landstreet J. D., et al. 2002, A\&A A 392, 637

Shulyak, D., Ryabchikova, T. A., Mashonkina, L., \& Kochukhov, O. 2009, A $\mathscr{\&} A$ 499,879

Sousa J. C., \& Cunha M. S. 2008, Contrib. Astron. Obser. Skalnaté Pleso 38, 453

Théado, S., Dupret, M.-A., Noels, A., \& Ferguson, J. W. 2009, A\&A 493, 159

Vauclair, S., \& Théado, S. 2004, A\& A 425, 179

Wade, G. A.; Aurire, M.; Bagnulo, S.; Donati, J.-F. et al. 2006, A $\mathscr{G} A$ 451, 293 